techniques/strategies; Describe relationships among patient characteristics (including biomarkers), symptoms/QoL, and adaptive techniques/strategies. METHODS: Fifteen patients $>18 \mathrm{yrs}, \mathrm{KPS} \geq 70 \%$, and $\leq 2$-months post Grade II-LGG diagnosis were enrolled. FACT-G/Br/Cog, Beck-DepressionInventory (BDI)-II, FACIT-fatigue, distress-scale were completed at 2, 4, and 6-months post-diagnosis. Qualitative interviews identifying symptoms/challenges and adaptive techniques/strategies were conducted to code themes at 4 and 6 months. Trajectory of change in symptoms/QOL was described using means and $95 \%$ confidence intervals. RESULTS: Mean age $=40,27 \%$ male, $93 \%$ white, $40 \% \mathrm{KPS} \geq 90 \%, 67 \%$ gross total resection; majority $(67 \%)$ with frontal tumors. Pathology included diffuse astrocytoma/infiltrating glioma $(60 \%)$ and oligodendroglioma $(40 \%), 1 \mathrm{p} 19 \mathrm{q}$-codeletions $(40 \%)$, IDH1mut (60\%), and TERTmut (53\%). Mean QoL scores at 2,4,6 months, respectively were FACT-Brain-Subscale (0-76):50,50,54; FACT-BR $(0-184): 126,126,130 ; \quad$ FACT-Cog-impairment-subscale $\quad(0-72): 50,49,46$ BDI-II (0-63): 15.2,16.5,13.9; Distress (1-4):2.2,1.5,1.8. FACIT-Fatigue scores $(0-52): 31,32,35$ suggest a clinically important decrease in fatigue $(+/$ 3). Initial analyses suggest that patients with either IDH1mut, TERTmut consistently reported lower QoL and higher distress, depression, fatigue scores. Seizures were reported with IDH1mut $(11 \%)$ but not IDH1wildtype. Lower QoL, depression, fatigue were seen with $1 \mathrm{p} 19 \mathrm{q}$-codeletions. Males reported higher QoL and lower depression/fatigue. Qualitative interview data will be presented identifying sustainable adaptive strategies in association with characteristics, symptoms and challenges. CONCLUSIONS $1 \mathrm{p} 19 \mathrm{q}$-codeletions and IDH1/TERTmut may be related to lower QoL (from IDH1mut-related seizures). Future studies to explore 1p19q-codeletions/ IDH1/TERTmut subtypes as predictors for QoL/adaptability to symptoms/ challenges should be conducted.

\section{QLIF-35. EFFECTS OF INTEGRATIVE THERAPIES ON DISTRESS, PAIN, AND FATIGUE IN PATIENTS WITH PRIMARY BRAIN TUMORS: PRELIMINARY RESULTS FROM AN INTEGRATIVE MEDICINE REGISTRY \\ Laura Donovan ${ }^{1,2}$, Angelica Rusilowski ${ }^{3}$, Katherine Taromina ${ }^{1}$} Teri Kreis ${ }^{1,2}$, Mary Welch ${ }^{1,2}$, Andrew B. Lassman ${ }^{1,2}$, Elena Ladas ${ }^{1}$ and Fabio Iwamoto 1,$2 ;{ }^{1}$ Columbia University Medical Center, New York, NY, USA, ${ }^{2}$ The Neurological Institute of New York, Columbia University, New York City, NY, USA, ${ }^{3}$ Columbia University, Mailman School of Public Health, New York, NY, USA

Integrative medicine therapies are utilized by up to $80 \%$ of cancer patients for a variety of reasons, including symptom management and improvement in quality of life. There has been an increase in research with conflicting data on the efficacy of integrative therapies in cancer patients. In particular, there is a paucity of data regarding use of integrative therapies and their potential impact on symptoms and quality of life in patients with primary brain tumors. We report preliminary outcomes from an integrative medicine program available free of cost to neuro-oncology patients at Columbia University Medical Center (CUMC). 24 patients participated in an IRBapproved integrative therapies registry. Patients completed questionnaires pre- and post-integrative therapy sessions regarding symptoms including distress, pain, nausea and vomiting, and fatigue. There were 14 women and 10 men with a median age of 53.5 years (range $33-72$ years) and $63 \%$ with a diagnosis of glioblastoma. 21 patients self-identified as non-Hispanic white $(88 \%)$. The most common integrative therapies provided included acupuncture, acupressure, massage, and aromatherapy. Meditation, reiki, qi gong, and yoga were also offered. We compared pre- and post-intervention surveys for symptoms of distress ( $\mathrm{N}=33$ pre, 32 post), pain ( $\mathrm{N}=20$ pre, 15 post), and fatigue ( $\mathrm{N}=15$ pre, 8 post). The mean scores decreased for: distress (scale $0-10)$ from 5.79 to $1.84(\mathrm{p}<0.0001)$, pain (scale $0-4)$ from 2.15 to 0.47 $(\mathrm{p}<0.0001)$, and fatigue (scale $0-4)$ from 1.93 to $1.5(\mathrm{p}=0.17)$. These results suggest that integrative therapies may improve perceptions of distress and pain in patients with primary brain tumors. Additional research focusing on the effect and duration of specific treatments and their impact on overal quality of life is needed in this patient population.

\section{QLIF-36. ROLE OF NURSES FOR THE IMPLEMENTATION OF TTFIELDS IN DAILY CLINICAL ROUTINE IN GLIOBLASTOMA TREATMENT}

Mandy Kilias ${ }^{1}$ and Cristina Pellet ${ }^{2} ;{ }^{1}$ Department of Neurosurgery, Charité University Hospital Berlin, Berlin, Germany, ${ }^{2} \mathrm{CHUV}$, Lausanne University Hospital, Neurooncology, Department of Clinical Neurosciences, Lausanne, Switzerland

INTRODUCTION: Glioblastoma multiforme (GBM) is the most common type of primary malignant brain tumor in adults and the diagnosis is devastating to patients and their families. Treatment includes many modalities such as surgery, radiation, chemotherapy and tumor treating fields (TTFields). The latter is a new therapy involves placing transducer arrays that emit alternating electric fields on the shaved scalp to interfere with tumor cell division. Personalizing best treatment decision to achieve optima therapy efficacy requires profound and extensive patient education. In this context, it is of critical value to have oncology nurses as an integral part of the therapy algorithm. METHODS: To implement TTFields into GBM therapy we critically analyzed our current treatment pathway to find out where nurses can improve interactions with GBM patients. In addition, we performed a quality assessment of specific wording used to explain TTFields to the patients to guide them through TTFields therapy. Specific training of nurses to educate patients on how to integrate TTFields therapy into their daily work-life increases therapy acceptance and compliance. RESULTS: We successfully assessed how nurses can integrate patient education into the treatment pathway. Starting at diagnosis and further on during radiochemotherapy, we present all treatment options including TTFields repeatedly in a patient adapted way. This allows patients to make conscious treatment decisions that ensure acceptance and compliance. In addition the integration of nurses in the treatment team reduced the physicians' daily work load. CONCLUSIONS: Specific TTFields training for nurses fundamentally shaped the understanding of the therapy and subsequently improved patient education, acceptance and compliance. This procedure reduces physician's work load, increases motivation of nurses and improves therapy efficacy to deliver the best treatment to patients with GBM. We experienced benefits and therefore suggest implementing trained nurses into the GBM therapy algorithm.

QLIF-37. SURVIVORSHIP IN BRAIN METASTASES PATIENTS: THE ROLE OF THE NEURO-ONCOLOGY TEAM IN ADDRESSING EDUCATIONAL NEEDS IN NON-NEURO-ONCOLOGY PROFESSIONALS?

Catherine McBain $^{1}$, Sara Robson ${ }^{1}$, Elizabeth Molloy ${ }^{1}$, Julie Emerson ${ }^{1}$, Charlotte Smith $^{1}$, James Leggate ${ }^{2}$, Rovel Colaco ${ }^{1}$ and Gillian Whitfield ${ }^{1}$; ${ }^{1}$ The Christie NHS FT, Manchester, United Kingdom, ${ }^{2}$ Salford Royal Foundation Trust, Manchester, United Kingdom

BACKGROUND: Outcomes of patients treated for brain metastases are improving, with growing numbers of long-term survivors following surgery and stereotactic radiosurgery (SRS). Most patients continue longer-term care predominantly under their site-specific oncology team (breast, lung etc). It was proposed that survivorship in brain metastases patients might be enhanced by addressing neuro-oncology-specific educational needs in less specialised health professionals. AIMS: To identify educational needs of health care professionals caring for patients following treatment for brain metastases. To provide a comprehensive educational package to meet needs identified. METHODS: Educational needs were evaluated via a baseline survey addressing confidence in managing neuro-oncology problems. The questionnaire was electronically disseminated to an unselected, diverse group of health professionals including primary care physicians, nurses and allied health professionals in hospital and community settings. The results were collated to inform the content of the training. Surveys of attendees' confidence in managing aspects of care were completed at registration, end of day and 5 weeks later. A two-sample Wilcoxon rank-sum (Mann-Whitney) test was applied to compare the data RESULTS: 266 responses were received, although as it was disseminated electronically, response rate cannot be calculated. A whole-day training event was designed around the responses. Topics covered the acute and late toxicity of brain metastases treatments, management of epilepsy, emergencies and the role of rehabilitation in preserving function and enhancing quality of life. Results from the 90 attendees showed statistically significant improvement in confidence above baseline across all aspects at both time-points (end of day and 5 weeks later), suggesting that the attendees benefitted on the day and that benefit was retained. CONCLUSIONS: Significant unmet educational need was demonstrated in the confidence of non-neuro-oncology professionals caring for brain metastases patients. Targeted educational intervention achieved durable, measurable improvement. Further work to addressing survivorship in brain metastases populations is warranted.

\section{QLIF-38. TUMOR TREATING FIELDS FOR THE TREATMENT OF GLIOBLASTOMA MULTIFORME IN DAILY PRAXIS: EXPERIENCE FROM TWO HOSPITALS IN GERMANY}

Stephanie May ${ }^{1}$, Julia Goebel ${ }^{1}$, Rüdiger Gerlach ${ }^{2}$ and Dietmar Krex ${ }^{1}$;

${ }^{1}$ University Hospital Carl Gustav Carus TU Dresden, Department of Neurosurgery, Dresden, Germany, ${ }^{2}$ Helios Hospital Erfurt, Department of Neurosurgery, Erfurt, Germany

INTRODUCTION: A recent clinical trial combining tumor treating fields (TTFields) with adjuvant temozolomide in patients with newly diagnosed glioblastoma multiforme (GBM) demonstrated a significant increase in progression-free (PFS) and overall survival (OS). The aim of our surveillance study was to examine the implementation of this new therapy approach in clinical practice and to analyze the acceptance and compliance. METHODS Patients with a diagnosed GBM of two independent centers in Germany were treated with TTFields (Optune () ). TTFields are applied via four transducer arrays and are generated by a portable device. Study data indicate the therapy compliance supposed to be above $75 \%$. We created a questionnaire that covers handling of the device, side effects, overall satisfaction and if they 
would recommend the therapy. Additionally, we analyzed the relationship of PFS and therapy compliance. RESULTS: During an observation period of 15 months, 21 patients received TTFields. More patients will follow. Twelve patients treated with TTFields took part in the survey. All patients were very satisfied with technical support and supply. Most patients reported easy handling in everyday life $(58 \%)$, the remaining patients perceived some limitations. Side effects were limited to skin irritation $(50 \%)$, sleep disturbance and back pain ( $25 \%$ of the patients). Around $63 \%$ had a recommended compliance. Within the observation period $29 \%$ died, $10 \%$ showed progressive and $71 \%$ showed stable disease. Although several patients described limitations in everyday life, $83 \%$ would decide on TTFields treatment again. CONCLUSION: The survey of patients with ongoing TTFields treatment in two independent centers revealed a good therapy acceptance and compliance. The PFS tends to approach the published data. Although patients reported of handling difficulties and side effects, most patients could integrate the therapy in their everyday life and would decide on the treatment again.

\section{QLIF-39. BURNOUT AMONG NEURO-ONCOLOGY PROFESSIONALS \\ Shlomit Yust-Katz ${ }^{1}$, Barbara J. O’Brien ${ }^{2}$, Alvina A. Acquaye ${ }^{3}$, Elizabeth Vera ${ }^{3}$ and Terri Armstrong ${ }^{3} ;{ }^{1}$ Davidoff Cancer Center and Tel-Aviv University, Tel-Aviv, Israel, ${ }^{2}$ Department of Neuro-Oncology, University of Texas MD Anderson Cancer Center, Houston, TX, USA, \\ ${ }^{3}$ Neuro-Oncology Branch- NIH, Bethesda, MD, USA}

BACKGROUND: Burnout is a syndrome characterized by emotional exhaustion, depersonalization, and loss of meaning or purpose in work. CNS tumors are highly aggressive tumors with very poor prognosis. Caring for patients with CNS tumors can be challenging, and may place neuro-oncology health care providers at risk for burnout. The goal of this study was to explore the incidence and associated factors for burnout in neuro-oncology health care professionals. METHODS: A web-based survey of neuro-oncology professionals was conducted between September and December 1, 2016. The survey included the Maslach Burnout Inventory, a 22-item questionnaire considered the gold-standard tool for measuring professional burnout. In addition, questions on demographic, career and lifestyle features were included. RESULTS 427 neuro-oncology professionals participated in the survey. Participants were primarily female $(54 \%)$ physicians $(72 \%)$ practicing in the United States $(70 \%)$ and a median age between $35-44$ years. Although only $32 \%$ reported experiencing burnout currently, $63 \%$ of participants were identified as having high burnout by the MBI-HS, with basic scientists having the highest overall rate $(83 \%)$. Remarkably, only $19 \%$ reported that their institution had a mechanism in place to address the prevention or support for burnout. More than $70 \%$ of physicians reported working more than 50 hours per week, and $95 \%$ reported completing work-related tasks at home. Nearly half of participants reported significant stress (7-10 in a scale of 0-10), and did not meet exercise and sleep recommendations for a healthy lifestyle. $55 \%$ of respondents considered their income not adequate for the time and effort they devote to their profession. CONCLUSION: Burnout among neuro-oncology health care providers is a common problem. Estimating the prevalence of burnout and associated risk factors is imperative for improving professional and personal satisfaction and patient care. Implantation of burnout preventive strategies should be considered in the future.

QLIF-41. UNDERSTANDING EMOTIONAL DISTRESS IN PRIMARY BRAIN TUMOR PATIENTS: IS THERE A RELATIONSHIP WITH FEAR OF DEATH?

Ashlee Loughan ${ }^{1}$ and Sarah Braun ${ }^{2} ;{ }^{1}$ Division of Neuro-Oncology- Virginia Commonwealth University, Richmond, VA, USA, ${ }^{2}$ VCU Health / Massey

Cancer Center, Richmond, VA, USA

BACKGROUND: Learning of a terminal diagnosis may lead to emotional distress including symptoms of depression and anxiety. In fact, depression prevalence rates are as high as $40 \%$ in brain tumor patients. Although quality of life has received increased interest in cancer populations, little is known about brain tumor patients' underlying fear of death and its potential relationship to overall emotional distress. METHODS: Fifty-one patients ( $51 \%$ male, Mage $49 \mathrm{yrs}$, Medu $15 \mathrm{yrs}$ ) diagnosed with primary brain tumors participated in routine neuropsychological evaluations including emotional questionnaires: Beck Anxiety Inventory (BAI) and Beck Depression Inventory $2^{\text {nd }}$ edition (BDI-II). A single-item question from the BAI assessed fear of dying. Descriptive analyses, t-tests, and Pearson correlations were conducted to explore the relationship between patients' fear of dying and overall symptoms of depression and anxiety. For analyses of overall anxiety symptoms, the single-item question assessing fear of dying was removed to reduce multicollinearity. RESULTS: Thirty-five percent $(n=18)$ of patients reported fear of dying at the time of evaluation. Anxiety scores (BAI) were significantly higher for patients who reported a fear of dying $(\mathrm{M}=16.56, \mathrm{SD}=11.258)$ when compared to those who did not $(\mathrm{M}=9.00, \mathrm{SD}=8.895) ; \mathrm{t}(49)=-2.637, \mathrm{p}=.011$. There was also a positive correlation between the severity of fear and overall depression (BDI-II; $\mathrm{r}=.294, \mathrm{p}=.036$ ) and anxiety scores (BAI; $\mathrm{r}=.549, \mathrm{p}=.000$ ). All descriptive statistics and relationships between variables will be presented. CONCLUSIONS: Fear of dying was high in this sample of brain tumor patients. Patients who reported this fear had higher levels of anxiety and depression than those who did not. This was demonstrated between groups and when exploring fear severity levels. This research provides insight into the distress experienced by brain tumor patients, which may lead to more effective emotional interventions and improved quality of life.

\section{QLIF-42. INCIDENCE AND CHARACTERIZATION OF}

DERMATOLOGIC ADVERSE EVENTS IN PATIENTS TREATED WITH TUMOR TREATING FIELDS AND IMPACT ON OVERALL SURVIVAL

Ashley Sumrall, Serena Perera, Daniel Haggstrom, David Jennings,

James Symanowski and Myra Robinson; Levine Cancer Institute,

Charlotte, NC, USA

TTFields interfere with glioma cell division and are delivered via arrays applied to the scalp. Recommended treatment for glioblastoma involves administration of TTFields at a daily compliance rate of $75 \%$ (18 of 24 hours) to prolong OS. Given that the primary adverse effect of TTFields involves skin changes, disruptions in treatment may result from dermatologic adverse effects (dAE). Prior studies reveal an incidence of dAE between $16-43 \%$, but they are poorly categorized. Little is known about time to onset or duration of dAE. We reviewed a cohort of patients $(n=33)$ treated with TTFields between 2014-2017. Median duration of treatment with TTFields was 5.3 months (1.1-19.4). We identified 10 episodes of contact dermatitis, 8 erosions, 4 cases of folliculitis, and 2 ulcerations. Median time to onset of dAE was 69 days (13-123). Duration of dAE varied depending on subtype, but median duration was 64 days (13-397). Of 13 patients who developed dAE, 4 $(30.8 \%)$ had a delay. Median treatment time with TTFields was 1815 hours $(86$ - 9446) with median daily compliance of 11.3 hours per day (0.5-22.1). Median OS of entire cohort was 8.7 months $(95 \%$ CL: 7.0 - 12.1 months). Threshold analyses using Cox proportional hazards regression identified the best cutpoint for cumulative hours of treatment associated with OS. This value was used to evaluate association with incidence of dAE. Per 1000 hour increase in hours worn, HR $=0.812$ The optimal cumulative hours worn identified for OS benefit is 2987 hours. The risk of death in those with at least 2987 cumulative hours worn is 0.208 times the risk of death in those with fewer hours worn. Of 13 patients with dAE, $4(66.7 \%)$ received greater than 2987 total hrs. Comparatively, of 20 patients without dAE, only $2(10.0 \%)$ received greater than $2987 \mathrm{hrs}(\mathrm{p}=0.1824)$.

\section{QLIF-44. SLEEP DISTURBANCE IN ADULTS WITH PRIMARY BRAIN} TUMORS AND THEIR FAMILY CAREGIVERS

Lin Lin ${ }^{1}$, Lung-Chang Chien ${ }^{2}$, Kathryn Lee ${ }^{1}$, Steven Paul ${ }^{1}$ and

Christine Miaskowski ${ }^{1} ;{ }^{1}$ University of California, San Francisco, San

Francisco, CA, USA, ${ }^{2}$ University of Nevada, Las Vegas, Las Vegas, NV, USA

INTRODUCTION: While patients with brain tumors (BT) report sleep disturbances during radiation therapy (RT), no study has compared patients and their family caregivers (FCs). This study examined changes in total sleep time (TST) in patients and FCs during and after RT. METHODS: From a longitudinal study on fatigue, pain, and sleep disturbance during RT for various types of cancer, patients with BT and their FCs were selected for this analysis. They completed the initial assessment at simulation (baseline) and were followed weekly (7 times) during RT, then bi-weekly ( 4 times) and monthly ( 2 time points) after completion of RT. Sleep measures were obtained with wrist actigraphy over 48 hours at each time point. Data were analyzed using mixed models. RESULTS: Patients $(\mathrm{n}=13)$ were $50.6 \pm 16.8$ years old, primarily white $(69 \%)$, and female $(69 \%)$. FCs $(\mathrm{n}=7)$ were older $(64.8 \pm 6.8)$, primarily white $(86 \%)$, and male $(71 \%)$. Although baseline TST was similar (patients $=386 \pm 20 ; \mathrm{FCs}=384 \pm 27$ minutes), FCs slept significantly less than patients across time $(\mathrm{p}<.001)$, averaging $<7$ hours during RT. Patients' TST gradually increased over time during RT, but did not differ between any two consecutive time points. By week 6, patients slept significantly more $(457 \pm 23$ minutes) than baseline $(\mathrm{p}<.05)$. At the final 4 post-RT assessments, patients maintained $>7$ hours $(\mathrm{p}<.05)$ while $\mathrm{FCs}$ averaged $<6$ hours. CONCLUSIONS: Results are consistent with prior reports that patients sleep more as early as week 1 after the initiation of RT. FCs' sleep disturbance was associated with greater fatigue and less social support in other studies. Although FCs' TST did not differ over time from baseline, short sleep duration continued at 4 months post-RT. FCs need to be more cautious with daytime activities such as driving and may require additional assistance during this vulnerable period. Other sleep characteristics and demographic and clinical factors should be considered in future analyses. 